\title{
A Study on Statistical Modelling on Recovery Informatics of Covid19
}

\section{Jaydip Datta ( $\square$ deepjoy1972@gmail.com )}

Indian Institute of Chemical Engineers (CRC) https://orcid.org/0000-0002-0013-5269

\section{Method Article}

Keywords: Covid19, Hyperbolic fit, Rate law, Pearson coefficient, Second-order rate law, epidemiological parameters, Remdesivir modelling, Molecular bonding, Pharmacokinetic stability constant

Posted Date: November 11th, 2020

DOl: https://doi.org/10.21203/rs.3.rs-105206/v1

License: (9) This work is licensed under a Creative Commons Attribution 4.0 International License. Read Full License 


\title{
A STUDY ON STATISTICAL MODELLING ON RECOVERY INFORMATICS OF COVID19
}

\author{
Jaydip Datta * \\ *Indian Institute of Chemical Engineers ( CRC ) , J.U campus , 700032 , INDIA \\ ( Email : deepjoy1972@gmail.com )
}

\begin{abstract}
-
In this article the positive cases of Covid19 in India with effect from $7^{\text {th }}$ September, 2020 to

$25^{\text {th }}$ October, 2020 are analysed for statistical relevance . The scattered data are used to find out a model equation correlating two variables number of recovered Covid -patient with an interval of regular seven days. The best fit regression analysis shows a significant correlation of Pearson coefficient $(r)$ with standard error $(s)$ with a second-order rate law .The rate constant $(k)$ obtained from this model can be simulated to stability aspects of antiviral drug modelling like Remdesivir as an objective of this work .
\end{abstract}

Keywords - Covid19, Hyperbolic fit, Rate law, Pearson coefficient, Second-order rate law, epidemiological parameters, Remdesivir modelling, Molecular bonding, Pharmacokinetic stability constant .

\section{INTRODUCTION :}

The important statistical parameters used to explain an epidemiology like Covid19 better Known as mortality rate( MR ), case fatality rate (CFR), Infection fatality rate(IFR)[ 1,2 ]. The mathematical model like Susceptibity - Infectivity - Recovery ( SIR ) [ 3 ]used mostly to quantify the different epidemiological stages. The stages are namely viral inoculation, spreading of viral infection and finally recovery from Covid19. The statistical models from best fit data analysis also used to explain the above epidemiological parameters ( MR , CFR , IFR ) are nonlinear regression modelling like hyperbolic regression, nth order polynomial regression, MMF regression, logistic or sigmoid regression .

\section{OBJECTIVE :}

In this article the final stage of SIR modelling, Recovery rate is modelled by Hyperbolic regression to obtain a second order rate law. In the other way the rate constant $(\mathrm{K})$ is analogous to pharmacokinetic approach of Remdesivir modelling of chemo informatics.The combination of recovery rate of Covid19 and Remdesivir modelling is called recovery informatics may be considered as main objective $[4,6,8,9]$ of this work . 


\section{MATERIALS AND METHODS:}

1. Availability of data - a set of data ( Figure - I) of number of days with seven days interval vs number of recovered cases with effect from $7^{\text {th }}$ September $-25^{\text {th }}$ October, 2020 .
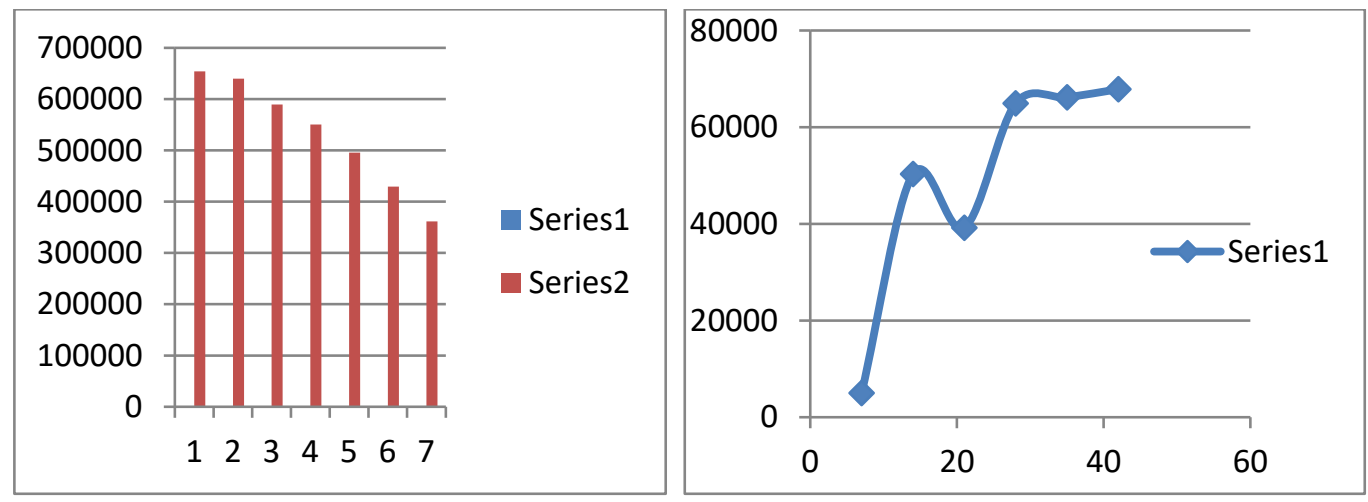

Figure -1 : Excell plot of scattered data ( 1 ) of number of days with seven days interval ( $x$ axis ) vs number of positive Covid-cases (y-axis ).

Active Covid cases with respect to Sept,7 to Oct ,25 -2020 in India (Sept7- $13,2020-6,45,014$, Sept14-20,2020 - 6,40,019, Sept 21-27,2020 -5,89,719, Sept 28-Oct 04 , 2020-5,50,545 , Oct 511,2020 -4,95,639 , Oct 12-18,2020-4,29,481 , Oct 19-25,2020-3,61,626 )- Dataset - ( D1 )

Retrieved from -Sharpest weekly fall as cases down 16\%, toll 19\% | Man hits docs over Covid death, -Oct 26, 2020, ethealthworld.com. (data report ) https://health.economictimes.indiatimes.com/?utm_source=Mailer\&utm_medium=-utm_medium--\&utm_campaign=--utm_campaign--

The above dataset is converted in terms of number of Recovered Covid-cases with respect to

Sept,7 to Oct ,25-2020 - [ ( 7,4995$)(14,50300)(21,39174)(28,64906)(35,66158)]$ -

dataset under subject of interest ( D2 ).

2. Data Informatics Tool : Standard Statistical Software Curve Expert v.1.4 .

RESULTS AND DISCUSSIONS: The results may be considered as output of the standard software analysed by a best fit regression methods to calculate the most important statistical parameters like model equation, Pearson coefficient $(r)$, standard error $(s)$, chi-sq relevance, covariance matrix and residual table.

The output of software after execution of dataset ( D2 ) 


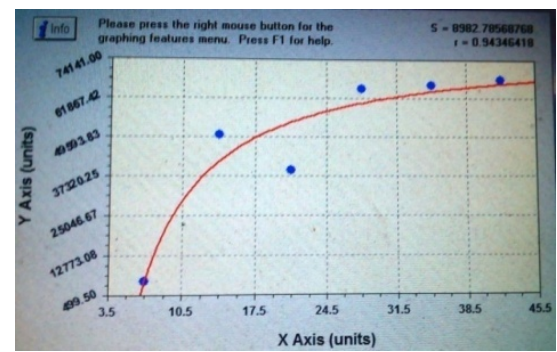

( Figure -II )

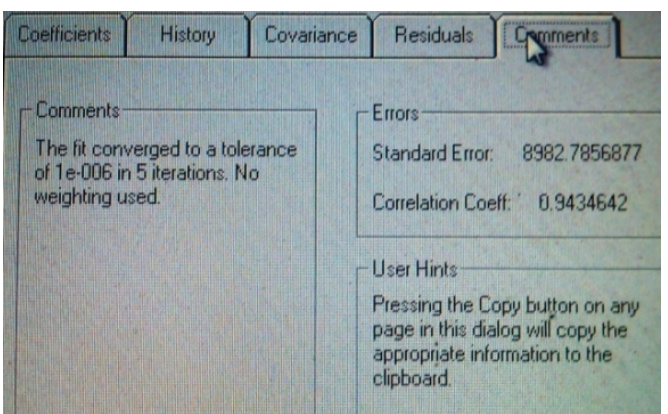
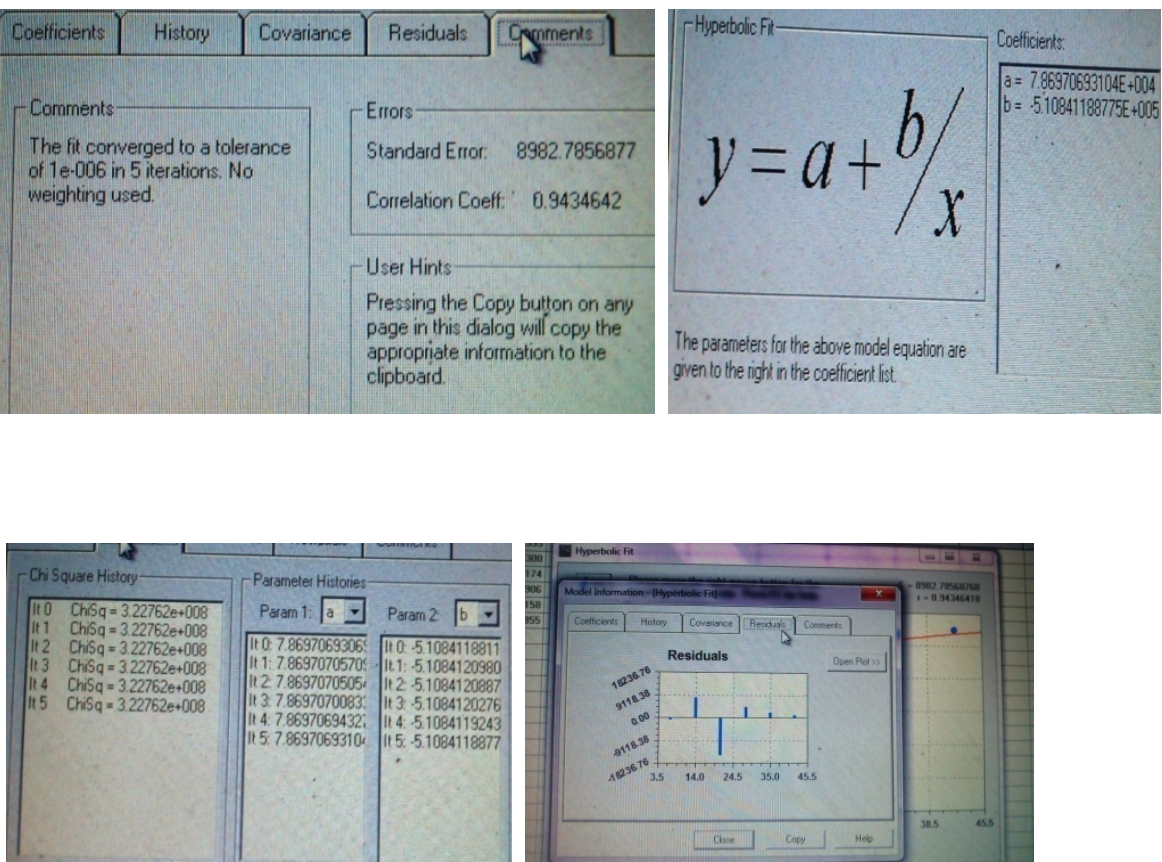

\section{Figure -II :}

The model rate equation [ time in days,$t=f$ ( number of recovered cases,$n$ ) can be executed as hyperbolic best fit( 2 ) as follows :

$$
\mathrm{t}=7.8697 * 10^{\wedge} 4-\left(5.1084 * 10^{\wedge} 5 / \mathrm{n}\right)
$$
( equation 1.0)

[ Where $a=7.8697 * 10^{\wedge} 4, b=5.1084 * 10^{\wedge} 5$ are called coefficient of hyperbolic fit with a 
covariance matrix $-2 \times 2$, equal residuals , $r=0.9464(0<r<1), S=$ standard error $=8983-1 * 10^{\wedge}(-6)$ iterative convergence ] [ 4 ].

Differentiating (equation 1.0 ) with respect to $\mathrm{n}$ we get -

$$
\begin{aligned}
d t / d n & =5.1084 * 10^{\wedge} 5 /\left(n^{\wedge} 2\right) \\
\text { or } \quad d n / d t & =\left[\left(n^{\wedge} 2\right) / 5.1084 * 10^{\wedge} 5\right] \\
d n / d t & =k\left(n^{\wedge} 2\right) \quad \text { (equation 2.0) }
\end{aligned}
$$

equation 2.0 represents the desired model equation pointing the rate of number of Covid-cases is directly proportional to square of number of recovered Covid-cases. obeying second-order rate law $[5,6]$. The second order rate constant $(k)=5 \cdot 1084 * 10^{\wedge} 5$ with a dimension of unit has been Expressed as only as time in day-number of covered patient ( $t-n$ ) kinetics [ 7 ] . The recovery rate can be co analysed with Remdesivir modelling as per (Figure - III) with equation 2.0 considering recovery informatics approach .

\section{CONCLUSION :}

The best fit statistical analysis of hyperbolic fit resumes a significant Pearson coefficient ( $r$ ), standard error $(\mathrm{s})$. The model equation 2.0 also represents a rate law showing a second -order relationship with a recovery rate constant or second order rate constant ( $K$ ) The scope of $K$ can be extrapolated to pharmacokinetic second order rate[8 ] law as follows :

Remdesivir ( D ) + Covid19 ( Nucleocapsid antigen ) $-----\rightarrow$ [ D-P Intermediate ] $---\rightarrow$ Drug Stability The stability constant of the Drug ( Keq ) $=[\text { D-P Intermedaie ] / [ ] }]^{*}[P]$ ( equation 3.0) the second -order rate law of the ( equation 3 ) can be expressed as :

$$
d C r / d t=K e q C p^{\wedge} \mathbf{2} \text { is equivalent to } d n / d t=k\left(n^{\wedge} \mathbf{2}\right)
$$
( equation 4.0 )

Where $\mathrm{Cr}=$ Molar Concentration of interaction for [ D-P Intermediate ] or Remdesivir - Covid19 stability interaction , $\mathrm{Cp}=$ Molar Concentration of [D-P Intermediate].

From (equation 4 )we can get ( Keq) Remdesivir - Protein [ D-P] equivalence of recovery rate constant $(k)$. From the pharmacological point use of molar equivalence of Remdesivir will enhance the recovery rate ( equation 2 ). Pharmacokinetic approach can be applied to chemo-informatics approach through molecular bonding] approach of drug design [ 9 ] as per following logic .

\section{MOLECULAR INFORMATICS STUDY [ 3 ] -}

A Combined Model for Covid19 (S-orbital ) + Remedesivir ( P-orbital ) ---------- $\rightarrow$

Covid -Remedesivir ( A SP Hybridized Model Hypothesis ) . 
The molecular model simulation K, Keq, molecular bonding approach of SP hybridisation

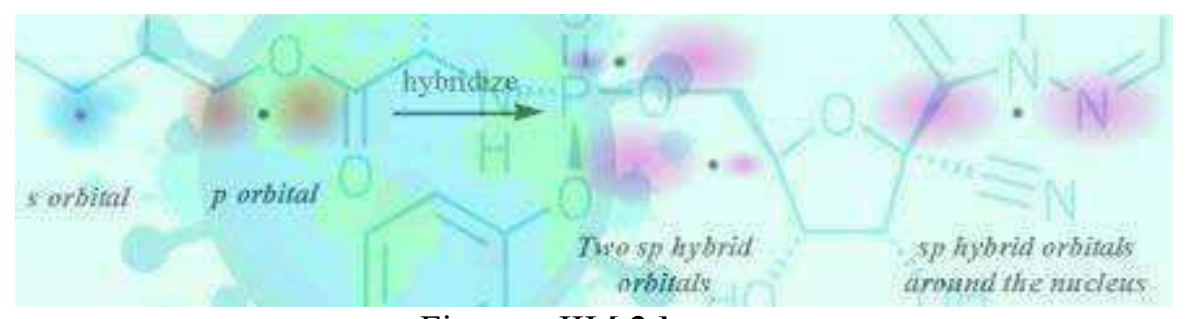

Figure - III [ 3 ]

The most important aspects of recovery informatics explains the use of an effective antiviral drug [ 10 ] in molar equivalence like Remdesivir ( Figure - III) will enhance the recovery rate ( equation 2.0 ) as usual .

\section{REFERENCE :}

1. COVID19 - A Correlation Study of Infection Fatality Rate vs Age, Jaydip Datta, DOI: https://doi.org/10.21203/rs.3.rs-85482/v2 .

2. Growth of D614G Virus : Morgan - Morgan - Finney ( MMF ) Regression, Jaydip Datta , DOI: https://doi.org/10.21203/rs.3.rs-92776/v1 .

3. Mathematical and Statistical Study on COVID19- SIR Model, Jaydip Datta, DOI: https://doi.org/10.21203/rs.3.rs-89766/v2 .

4.https://towardsdatascience.com/understanding-regression-using-covid-19-datasetdetailed-

analysis-be7e319e3a50.

5.https://arxiv.org/abs/2004.05684, kisor sahu , April ,2020.

6.https://www.medrxiv.org/content/10.1101/2020.05.29.20117069v1- May,2020 .

7. Kinetics methods for clinical epidemiology problems, Proc Natl Acad Sci U S A. 2015

17; 112(46): 14150-14155.Published online 2015 Nov

2. doi: 10.1073/pnas.1510927112,

PMCID: PMC4655575 ,PMID: 26578757 , Chemistry, Medical Sciences .

8.

https://en.wikipedia.org/wiki/Goodman \%26 Gilman\%27s The Pharmacological Basis of Therapeutics.

9. https://www.pdfdrive.com/burgers-medicinal-chemistry-drug-discovery-vol-3e20346098.html

10. COVID19 DRUG REVIEW DISCUSSIONS (Version Version 1.0). Zenodo, http://doi.org/10.5281/zenodo.4244641, JAYDIP DATTA , 2020. 


\section{SUPPLIMENTARY LINK :}

1.https://www.academia.edu/44413464/SUPPLIMENTARY RESOURCES Health Statistics Applied T o Covid19 Recovery kinetics, Jaydip Datta .

2. JAYDIP DATTA. (2020). SUPPLIMENTARY Dataset - A STUDY ON STATISTICAL MODELLING ON RECOVERY INFORMATICS OF COVID19 (Version Version 1.0) [Data set]. Zenodo. http://doi.org/10.5281/zenodo.4255246

\section{CODE : JSON EXPORT -}

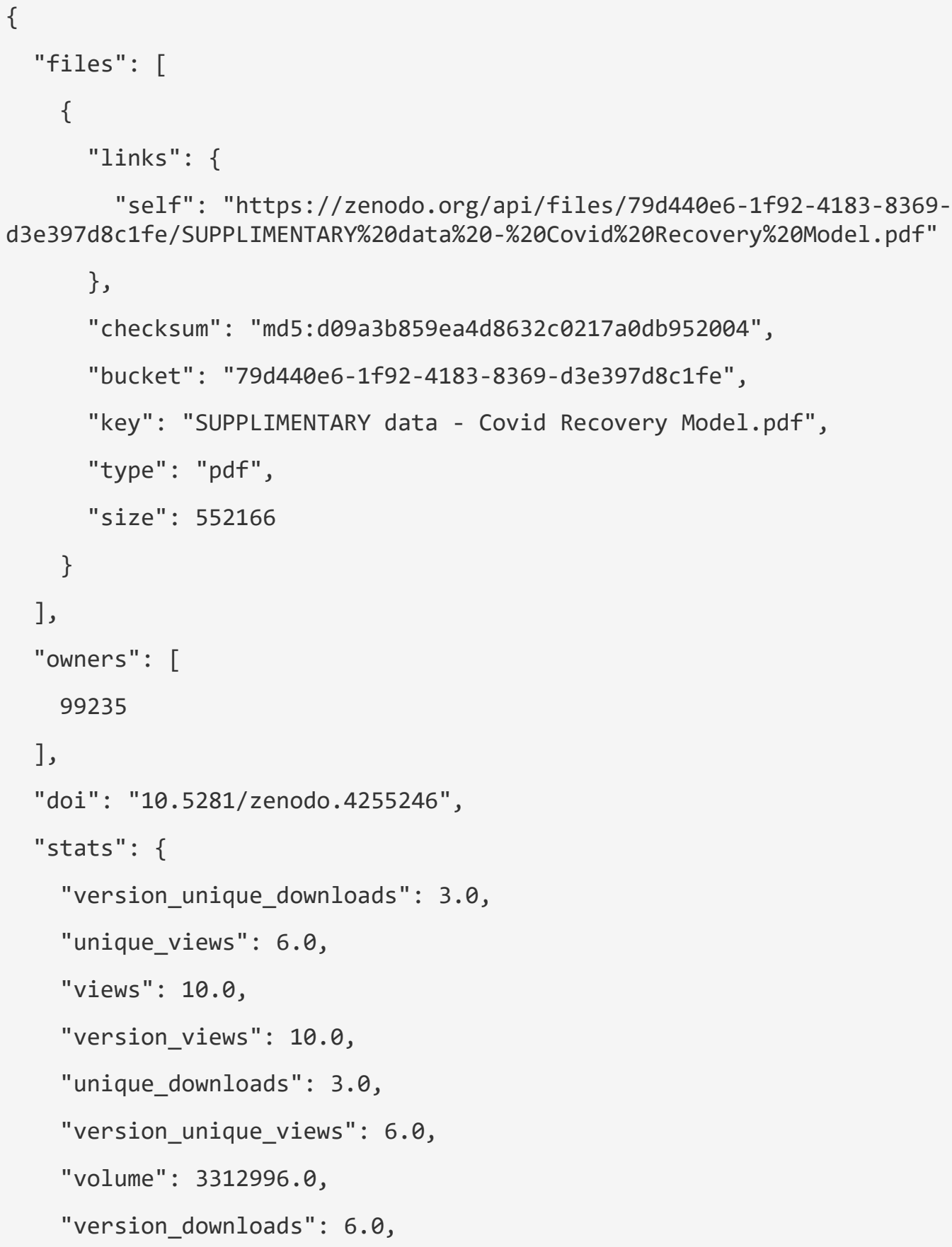




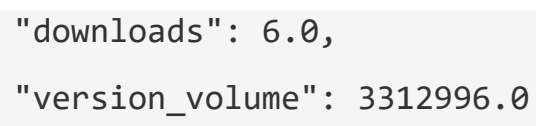

"description": "<p>Collection of data \&ndash; a set of data of number of days with seven days interval\&nbsp;vs number of recovered cases with effect from 7th September \&ndash; 25th October ,2020. Active Covid cases with respect to Sept, 7 to Oct , 25 -2020 in India .\&nbsp;Retrieved from -Sharpest weekly fall as cases down $16 \%$, toll 19\% | Man hits docs over\&nbsp;Covid death, -Oct 26, 2020, ethealthworld.com. ( data report). The above dataset is converted in terms of number of Recovered Covid-cases with respect .Data Informatics Tool : Standard Statistical Software Curve Expert v.1.4. $\langle/ p\rangle "$,

"language": "eng",

"title": "SUPPLIMENTARY Dataset - A STUDY ON STATISTICAL MODELLING ON RECOVERY INFORMATICS OF COVID19",

"license" : \{

"id": "CC-BY-4.0"

\} ,

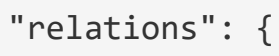




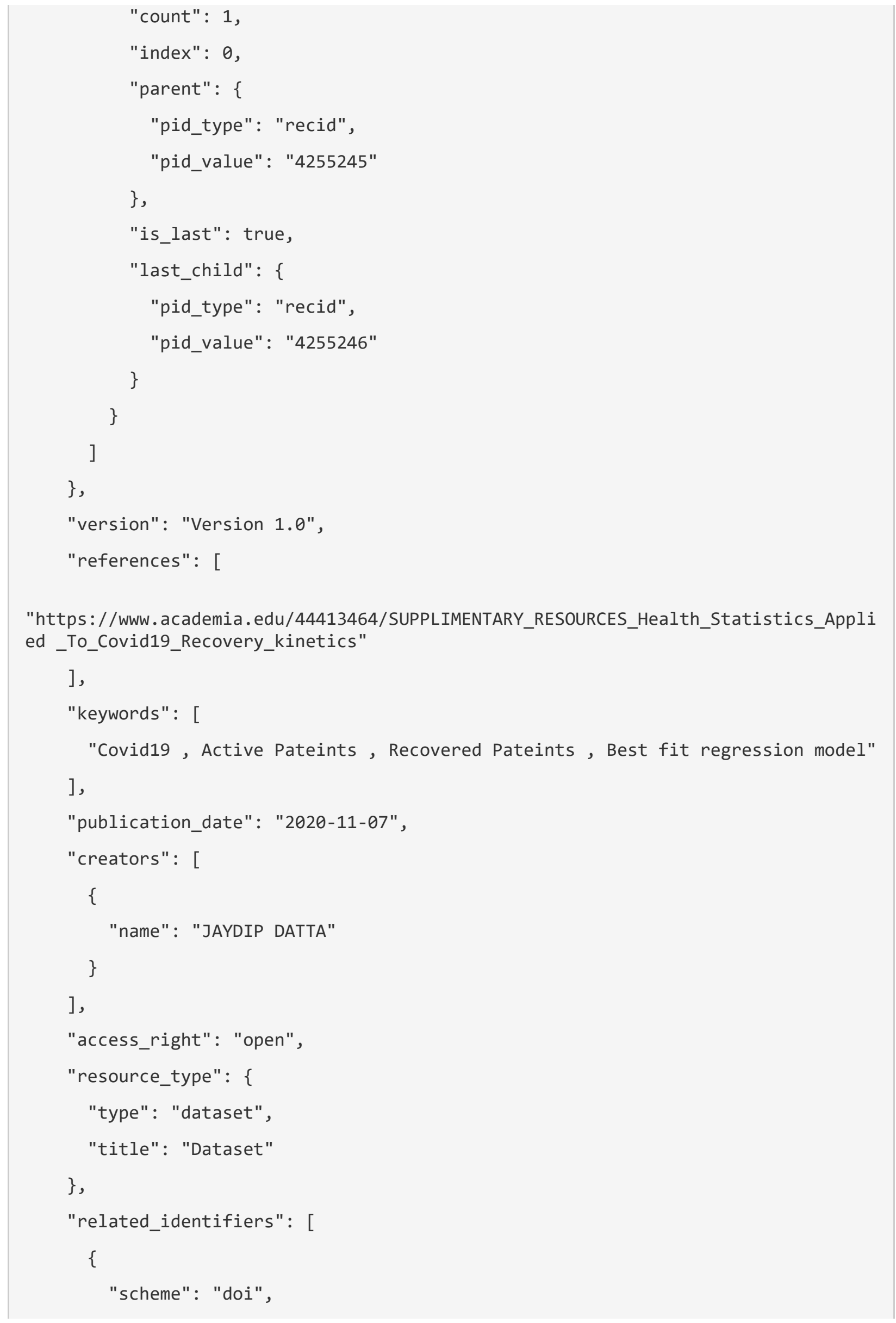




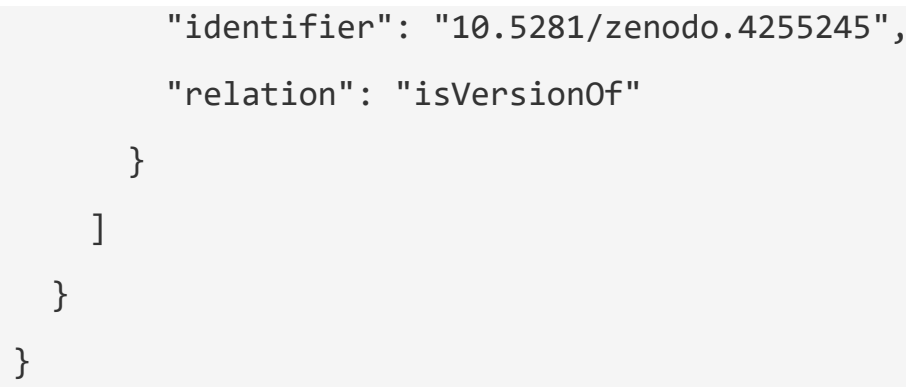

FUNDING STATEMENT: NOT AVAILABLE 

Figures
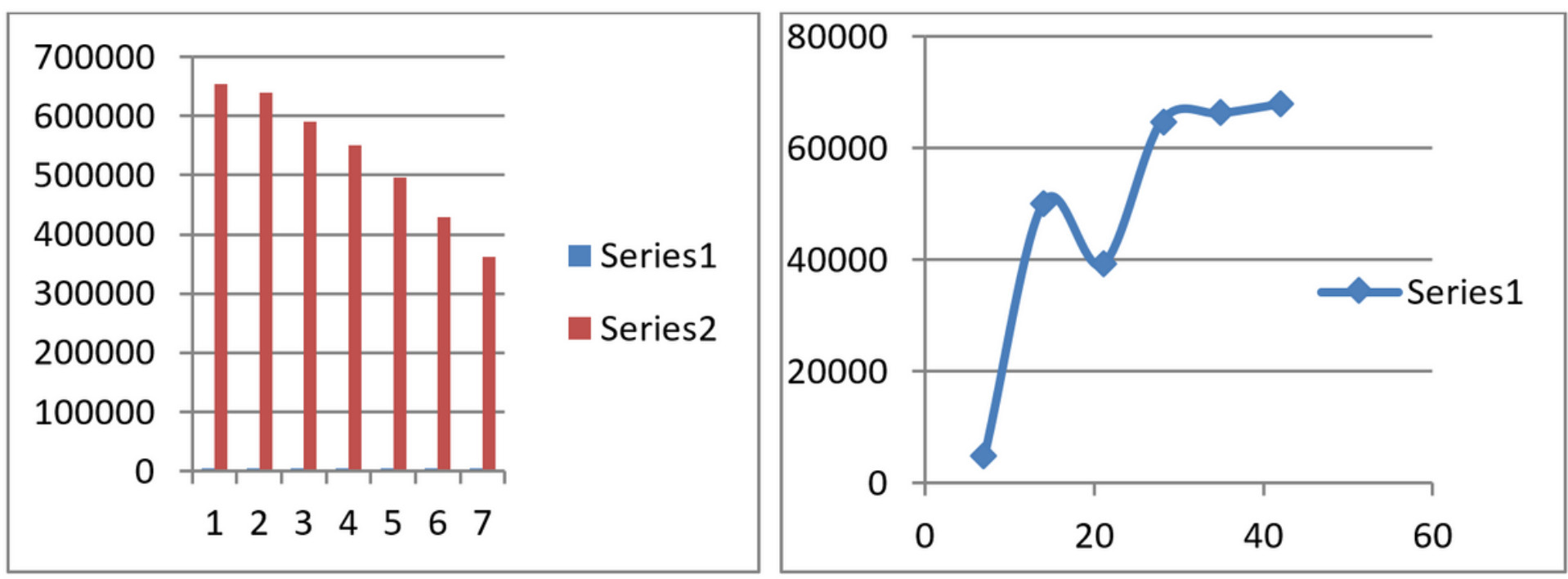

Figure 1

Excell plot of scattered data ( 1 ) of number of days with seven days interval ( $x$-axis ) vs number of positive Covid-cases (y-axis ) .
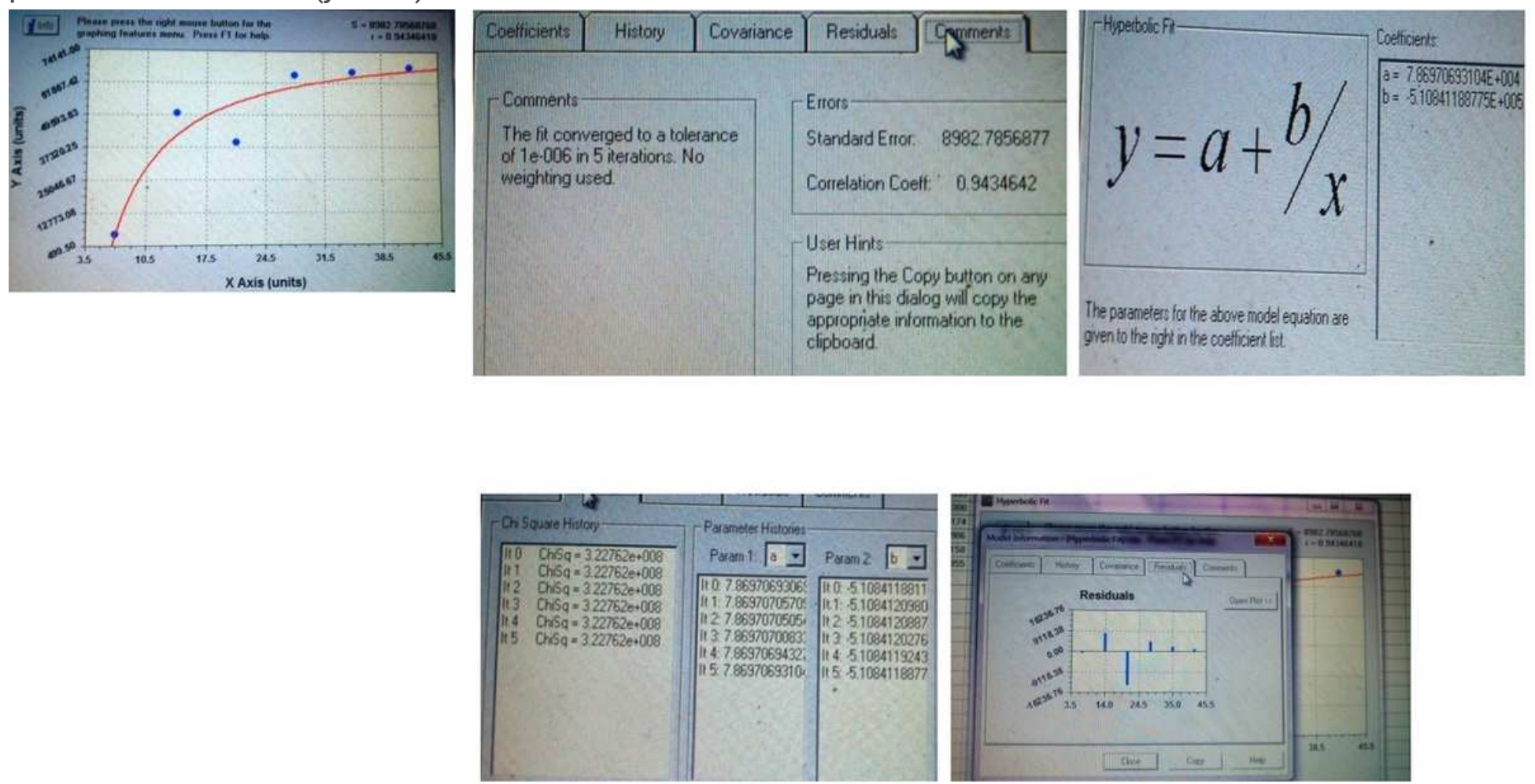

Figure 2

$\mathrm{t}$-axis - time in number of days, $\mathrm{n}$-axis - number of recovered cases in each seven days interval . 


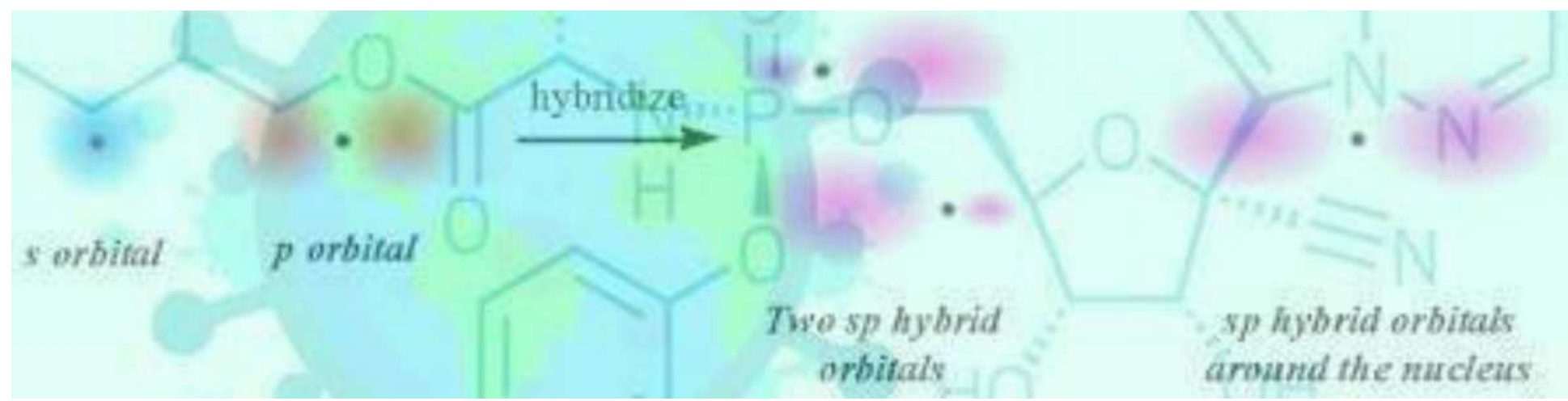

\section{Figure 3}

The molecular model simulation K, Keq , molecular bonding approach of SP hybridisation

\section{Supplementary Files}

This is a list of supplementary files associated with this preprint. Click to download.

- SUPPLIMENTARYdataCovidRecoveryModel.docx 